\title{
Recent results in reactions using radioactive ion beams
}

\author{
Elí F. Aguilera*† \\ Departamento de Aceleradores, Instituto Nacional de Investigaciones Nucleares, Apartado \\ Postal 18-1027, C. P. 11801, México, D. F., México \\ E-mail: eli.aguilera@inin.gob.mx
}

James J. Kolata

Physics Department, University of Notre Dame, Notre Dame, In, 46556-5670, USA

Ismael Martel, Angel M. Sánchez-Benítez, Luis Acosta

Departamento de Física Aplicada, Universidad de Huelva, E-21071 Huelva, Spain

\begin{abstract}
After more than two and a half decades of radioactive beam physics, the existence of nuclear halos remains as probably the most outstanding discovery in the field. Many measurements have been performed with light halo systems, especially with a ${ }^{6} \mathrm{He}$ projectile, which has a two-neutron halo. The total reaction cross sections that can be extracted from these measurements follow a systematic trend that can be compared to the behavior of the respective core, in this case ${ }^{4} \mathrm{He}$. By using an appropriate scaling for energies and cross sections, such comparison clearly shows the effects of the halo, which can be separated into static and dynamic effects. For a few systems, where the reactions of the halo have been explicitly measured, application of the same scaling criteria leads to the conclusion of a core-halo decoupling. The behavior of the proton-halo projectile ${ }^{8} \mathrm{~B}$ is similar to that of ${ }^{6} \mathrm{He}$ insofar as total reaction cross sections are concerned. The measured fusion data for ${ }^{8} \mathrm{~B}+{ }^{58} \mathrm{Ni}$, however, show a remarkable size effect of the halo which has no equivalent in the corresponding data for neutron-halo projectiles.
\end{abstract}

XXXIV edition of the Brazilian Workshop on Nuclear Physics

5-10 June 2011

Foz de Iguaçu, Parana state, Brasil

\footnotetext{
${ }^{*}$ Speaker.

${ }^{\dagger}$ Work partially supported by CONACYT.
} 


\section{Introduction}

Research with radioactive ion beams (RIBs) started nearly 25 years ago, when secondary beams of $\mathrm{He}$ isotopes were produced through projectile fragmentation of an energetic ${ }^{11} \mathrm{~B}$ primary beam, and then used to bombard different targets [1]. Since then, the respective beam production facilities have seen substantial improvements and many experiments with RIBs have been performed. The existence of nuclear halos, which was inferred soon after the first experiments, remains as probably the most outstanding discovery in the field. Halo nuclei, which can be considered as formed by a rather inert core nucleus surrounded by a diluted halo containing one or a few nucleons, have certainly attracted much attention. Different reaction mechanisms such as fusion, transfer and breakup have been studied, with interesting and sometimes astonishing results, as reviewed for instance in Refs. [2,3]. Measurements of the elastic scattering process, which is usually considered as a simple phenomenon, have also provided interesting new perspectives [4]. Within this context, the neutron-halo nucleus ${ }^{6} \mathrm{He}$ is probably the radioactive projectile that has been most studied.

Perhaps the simplest quantity that can be extracted from elastic scattering measurements is the total reaction cross section $\sigma_{R}$. An optical model analysis of the corresponding angular distributions yields this quantity, which is usually not very sensitive to the normal ambiguities in the optical potential. For data with normal experimental uncertainties, the respective values of $\sigma_{R}$ can be given with reasonable error bars that take into account the mentioned ambiguities. Recently [5], it was shown that the $\sigma_{R}$ results obtained for many systems with a ${ }^{6} \mathrm{He}$ projectile present a systematic behavior that can be contrasted with respective results for the corresponding core nucleus, ${ }^{4} \mathrm{He}$. The observed differences can be interpreted in terms of the halo feature. In addition, some experimental evidence that the interactions of the core and the halo do actually decouple from each other has also been presented [5,6]. The main purpose of the present work is to compile these recent results into a comprehensive study showing some of the most prominent effects of nuclear halos, especially the neutron-halo state of ${ }^{6} \mathrm{He}$. In addition, some very recent findings [7] about halo effects for the proton-halo projectile ${ }^{8} \mathrm{~B}$ will be also mentioned.

\section{Earliest evidence for static halo effects}

In the pioneering work of Tanihata et al. [1], several isotopes of He were used as projectiles, with an energy of nearly $800 \mathrm{MeV} /$ nucleon, to bombard targets of $\mathrm{Al}, \mathrm{C}$ and $\mathrm{Be}$. The interaction cross section $\sigma_{I}$ was measured, which is defined as the total cross section for the process of nucleon removal from the projectile. The authors defined also the interaction radius $\mathrm{R}_{I}$ through the expression $\sigma_{I}=\pi\left[\mathrm{R}_{I}(\mathrm{p})+\mathrm{R}_{I}(\mathrm{t})\right]^{2}$, where $\mathrm{p}$ stands for the projectile and $\mathrm{t}$ for the target, and from the data they were able to extract the respective $\mathrm{R}_{I}$ 's for the different projectiles. Fig. 1 shows the obtained results. It was found that the values of $\mathrm{R}_{I}$ for ${ }^{6,8} \mathrm{He}$ deviate from the simple $\mathrm{A}^{1 / 3}$ behavior. In particular, for ${ }^{6} \mathrm{He}$ the measured radius was about $35 \%$ larger than expected from the normal behavior, based on the value for ${ }^{4} \mathrm{He}$. These, along with similar results for a ${ }^{11} \mathrm{Li}$ projectile, gave the first hint for the existence of nuclear halos. Halo nuclei such as ${ }^{6} \mathrm{He}$ have unusually large radii, so we may say that the previous data show evidence for static effects of the halo, i.e., these effects are related only to the nuclear size. 


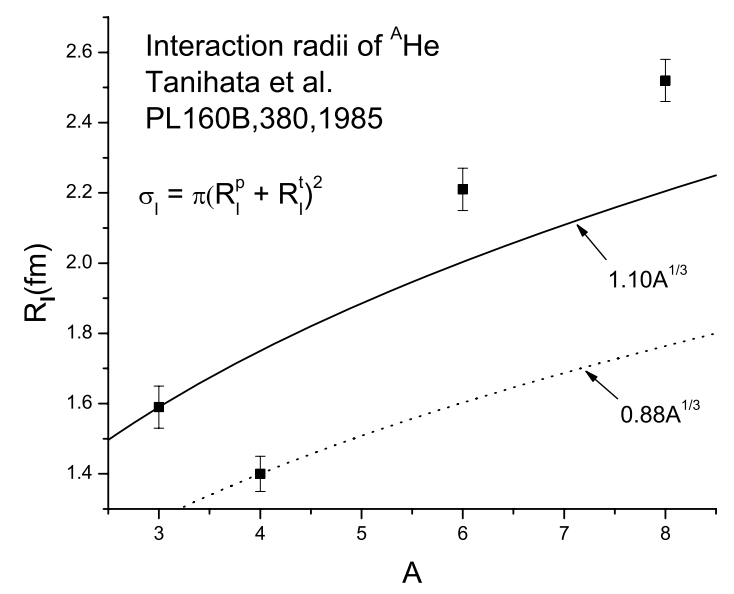

Figure 1: Interaction radii for He isotopes, from Ref. [1].

At lower energies, near the Coulomb barrier, dynamic effects of the halo are expected to appear. For the case of a ${ }^{6} \mathrm{He}$ projectile, for instance, if a heavy target is bombarded at energies low enough, the neutrons from the halo may have time to rearrange themselves in the force field of the target. In a semiclassical picture, we may think that the halo nucleus can get polarized in a Coulomb field strong enough, so that the neutral halo will touch the target before the core. This would increase the reaction cross section and the corresponding enhancement can be considered as a dynamic effect of the halo. Many measurements have been performed with ${ }^{6} \mathrm{He}$ projectiles at low energies. We now focus on this type of measurements.

\section{Summary of measurements for ${ }^{6} \mathrm{He}+{ }^{209} \mathrm{Bi}$}

The ${ }^{6} \mathrm{He}+{ }^{209} \mathrm{Bi}$ system is probably the halo system that has been most studied. Fig. 2 illustrates the respective processes that have been measured. After fusion, the compound nucleus cools down by evaporating neutrons. The evaporation residues are alpha emitters so the fusion yield can be determined by measuring the delayed alpha particles. In this way, the fusion cross sections were obtained for some near barrier energies [8]. Several direct processes can also occur in this system: the projectile can break up, leading to a final state where the target remains unchanged, with an alpha particle and two neutrons flying away; also, there can be a one neutron transfer to the target, in which case the remaining ${ }^{5} \mathrm{He}$ immediately decays into an alpha and a neutron, both of which fly away independently; finally, two neutrons can be transferred to the target, with an alpha particle flying away. All three processes produce at the end one alpha particle, so by counting these alphas the inclusive transfer-or-breakup (tr/bu) yield was determined $[9,10]$. This was done at several near and sub-barrier energies, where the elastic channel was also measured. Later works, where $\alpha$-n coincidences were measured, showed that $\sim 20 \%$ of the alpha's come from 1 n transfer [11], $\sim 55 \%$ come from $2 \mathrm{n}$ transfer [12], and $\sim 25 \%$ come from bu [13]. By fitting the elastic angular distributions within the framework of the optical model, the total reaction cross sections could be 


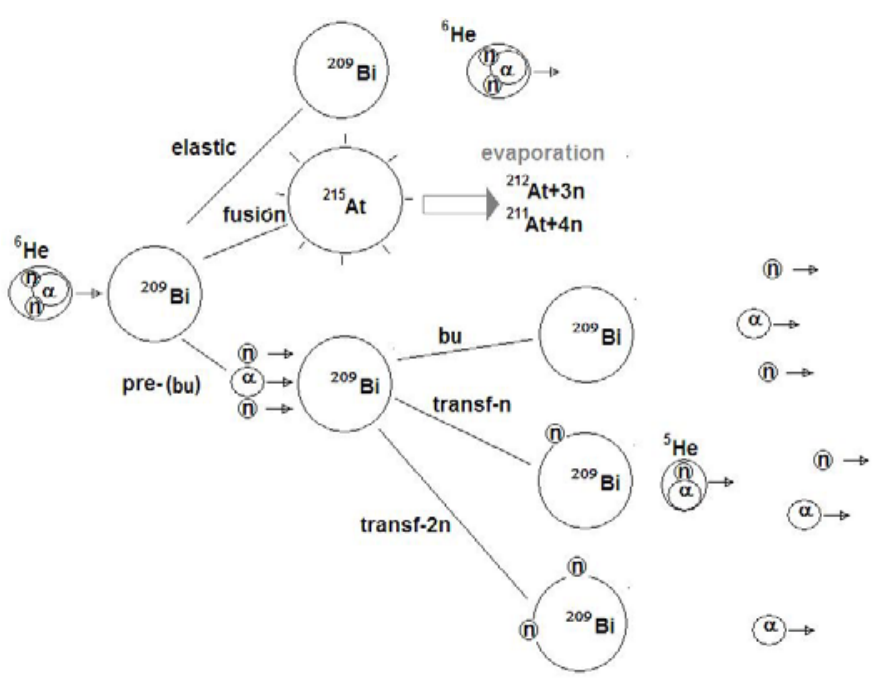

Figure 2: Schematic representation of the reaction mechanisms that have been measured for the ${ }^{6} \mathrm{He}+{ }^{209} \mathrm{Bi}$ system.

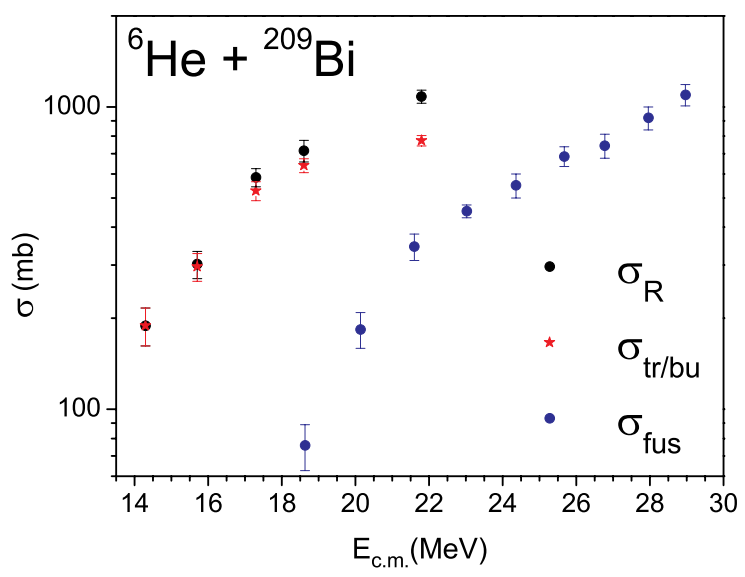

Figure 3: Fusion [8], transfer/breakup [9, 10], and total reaction cross sections [9, 10] for the ${ }^{6} \mathrm{He}+{ }^{209} \mathrm{Bi}$ system.

deduced. The results were consistent with the sum of fusion plus transfer/breakup, showing that these are in fact the dominant reaction channels for this system.

The results of the previous measurements are summarized in Fig. 3. Notice that the direct processes really dominate at low energies and almost saturate the total reaction cross section. This was an astonishing discovery since for normal systems all reaction channels tend to close down at these energies and, at most, only faint fusion yields remain. This enhancement phenomenon has been associated to the weak binding of the halo neutrons, i.e., it is a dynamic effect of the halo. This is consistent with the naive semiclassical picture mentioned above, whereas dipole polarizability might play an important role to enhance the reaction cross section. Large reaction cross sections also have been measured for other ${ }^{6} \mathrm{He}$ systems, as shall be seen in next section. 


\begin{tabular}{cccccc}
\hline target & process & lab & year & $\mathrm{E}_{\text {Red }} / \mathrm{V}_{\text {Red }}(\%)$ & Ref \\
\hline${ }^{209} \mathrm{Bi}$ & fus & & 1998 & & \\
& el, tr/bu & TwinSol-ND & 2000,2001 & $86,95,104,111,131$ & {$[9,10]$} \\
& 1 \& $2 \mathrm{n}$-tr, bu & & $2004,2005,2007$ & & {$[11,12,13]$} \\
${ }^{208} \mathrm{~Pb}$ & el, tr/bu & Louvain-la-Neuve & $2003,2008,2011$ & $159,82,94,105,129$ & {$[14,15,16]$} \\
${ }^{197} \mathrm{Au}$ & el & Louvain-la-Neuve & 2006 & 162 & {$[17]$} \\
${ }^{120} \mathrm{Sn}$ & el & Ribras-SP & 2010 & $143,149,163,169$ & {$[18]$} \\
${ }^{65} \mathrm{Cu}$ & el, tr, fus & SPIRAL-GANIL & 2008 & 267 & {$[19]$} \\
${ }^{64} \mathrm{Zn}$ & el, tr/bu, fus & Louvain-la-Neuve & 2004 & 114,155 & {$[20]$} \\
& fus & & 2006 & & {$[21]$} \\
${ }^{58} \mathrm{Ni}$ & el & TwinSol-ND & 2003 & 103 & {$[22]$} \\
${ }^{27} \mathrm{Al}$ & el & Ribras-SP & 2007 & $185,214,233,260$ & {$[23]$} \\
${ }^{12} \mathrm{C}$ & el & TwinSol-ND & 1991,1995 & $257,269,290$ & {$[24,25]$} \\
& el, reacts & Louvain-la-Neuve & 2004 & 526 & {$[26]$} \\
\hline
\end{tabular}

Table 1: $\sigma_{R}$ measurements with ${ }^{6} \mathrm{He}$ projectiles.

\section{Other $\sigma_{R}$ measurements for ${ }^{6} \mathrm{He}$ projectiles}

Table 1 shows the systems for which total reaction cross sections have been determined with ${ }^{6} \mathrm{He}$ projectiles. Nine different systems are distinguished, which are ordered from heavier to lighter targets in the Table, going from ${ }^{209} \mathrm{Bi}$ down to ${ }^{12} \mathrm{C}$. Since the reaction cross sections are usually deduced from elastic scattering measurements, the condition for the respective search was precisely that the elastic process should have been measured. Whenever other reaction channels have been additionaly measured, is indicated in the second column by writing the respective process. The measurements have been done at four different laboratories around the world, two of them in America (North and South America) and two in Europe. These are the TwinSol facility at the University of Notre Dame, where the first measurements were done, the facility at Louvain-la-Neuve, Belgium, the RIBRAS facility at the University of Sao Paulo, Brazil, and the SPIRAL facility at Ganil, France. As can be seen from the fourth column, it took two full decades to have the whole set of measurements, although most of them were performed during the last decade. The energies, given in the fifth column of the Table, refer only to the elastic scattering measurements, from which $\sigma_{R}$ values were determined. They are given in terms of a reduced energy that takes into account the respective differences in charge and mass; the reported numbers are actually a percentage of what we call the reduced barrier. Both the reduced energy and the reduced barrier will be defined below. In these units, it can be seen that the energy range of the whole data set goes from $\sim 80 \%$ up to $\sim 500 \%$ of the reduced barrier. So, the Table includes a set of ${ }^{6} \mathrm{He}$ systems with a large variety of target masses, covering a very large energy region. It is worth mentioning also that the data were taken by many independent groups, using different experimental techniques. It will be shown that the respective reaction cross sections follow a well defined systematic behavior.

In order to compare the data for the different systems, the recipes recommended by Gomes et al. [27] are used, i.e., the cross sections and energies are reduced according to the expressions 


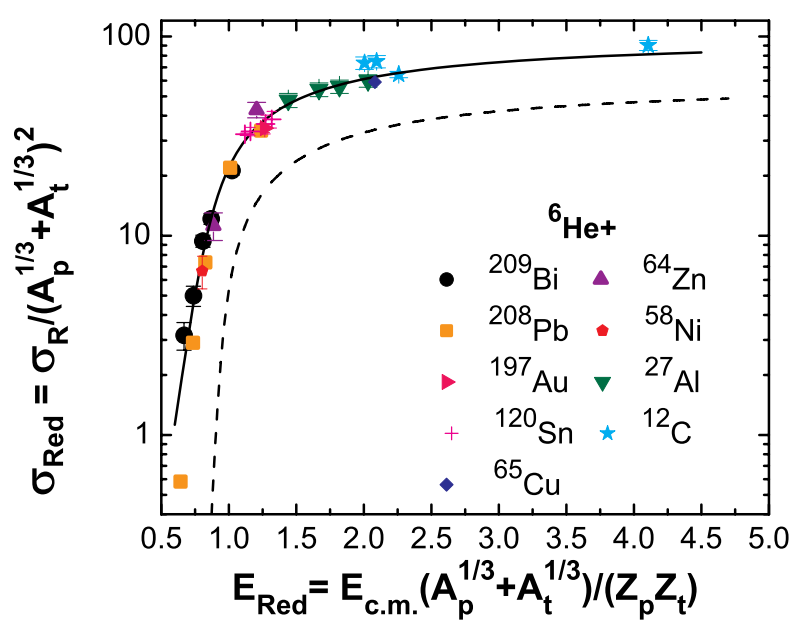

Figure 4: Reduced total reaction cross sections for the ${ }^{6} \mathrm{He}$ systems of Table 1. The solid line is a Wong function fit to the data, while the dashed line is the result of fitting similar data, but for ${ }^{4} \mathrm{He}$ projectiles (adapted from Ref. [5]).

$$
\sigma_{\text {Red }}=\frac{\sigma_{R}}{\left(A_{p}^{1 / 3}+A_{t}^{1 / 3}\right)^{2}} ; \quad E_{\text {Red }}=\frac{E_{c . m .}}{\left[Z_{p} Z_{t} /\left(A_{p}^{1 / 3}+A_{t}^{1 / 3}\right)\right]}
$$

In this way the effects of normal size changes, that go as $\mathrm{A}^{1 / 3}$, are taken into account, but size effects that deviate from this rule are not washed out. Of course, the sizes of halo nuclei do not follow this rule. The effect of the different nuclear charges is also taken into account in the energy scaling. Likewise, the reduced barrier is defined with the same scale factor as the reduced energy. With this definition, we may notice that the ratio $\mathrm{E}_{\text {Red }} / \mathrm{V}_{\text {Red }}$ which appears in the fifth column of Table 1 does actually coincide with the ratio $\mathrm{E}_{c . m .} / \mathrm{V}_{b}$, where $\mathrm{V}_{b}$ is the actual barrier height for each system. It is convenient to think in terms of reduced quantities because, as shall be seen below, all the systems of Table 1 can be characterized by a common value of $\mathrm{V}_{\text {Red }}$. When all the reaction cross section data corresponding to the previous table are reduced and plotted versus the reduced energy, an interesting picture is obtained.

Fig. 4 shows that, in spite of the wide mass and energy ranges, all systems fall along a well defined trajectory in this plot. This kind of alignment of experimental points was first noticed in Refs. [28, 29]. The trajectory can be nicely characterized by a reduced Wong function, which represents a one-dimensional Barrier Penetration Model calculation, but with reduced parameters [5]. Wong's formula in this case is written as

$$
\sigma_{\text {Red }}^{W}=\frac{\varepsilon_{0} r_{0 b}^{2}}{2 E_{\text {Red }}} \ln \left\{1+\exp \left[\frac{2 \pi}{\varepsilon_{0}}\left(E_{\text {Red }}-V_{\text {Red }}\right)\right]\right\} .
$$

The reduced radius $r_{0 b}$ is simply the barrier radius divided by $\left(A_{p}^{1 / 3}+A_{t}^{1 / 3}\right)$, and the barrier curvature parameter $\hbar \omega_{0}$ is reduced in the same way as the energy (see eq. 4.1 ) to obtain $\varepsilon_{0}$. The solid curve in Fig. 4 is a fit of eq. 4.2 to the experimental points, yielding the parameter values $\mathrm{V}_{\text {Red }}=0.780 \pm 0.014, \mathrm{r}_{0 b}=1.79 \pm 0.04, \varepsilon_{0}=0.43 \pm 0.06$ 


\section{Static and dynamic effects of the halo}

In order to elucidate the effects of the halo, a comparison was made with experimental data for reactions induced by ${ }^{4} \mathrm{He}$, which is the core of ${ }^{6} \mathrm{He}$. Fourty three values of $\sigma_{R}(\mathrm{E})$ were analyzed [5], for ${ }^{4} \mathrm{He}$ systems covering the same mass and energy ranges as those from Table 1 . The respective reduced points (not shown in the figure) also follow a well defined trajectory, which is very different from the previous one. Once again, the trajectory can be nicely characterized by a reduced Wong curve, shown by the dashed line in Fig. 4. The parameter values in this case are $\mathrm{V}_{\text {Red }}=0.913 \pm$ $0.005, \mathrm{r}_{0 b}=1.39 \pm 0.05, \varepsilon_{0}=0.175 \pm 0.006$. It can be seen that the reduced cross sections fall systematically lower in this case and the slope for fall-off at low energies is steeper.

By looking at the barrier parameters for the two Wong curves in Fig. 4, a big difference in the radii is observed. The reduced radius is nearly $30 \%$ larger for the ${ }^{6} \mathrm{He}$ systems and this is the main reason why the respective curve shifts up to higher cross sections. In fact, this ratio in the respective radii is similar to that observed by Tanihata et al. for the interaction radii of the same two isotopes (Fig. 1), despite the fact that they refer to conceptually different radii. Also, we can see that the barrier height is somewhat lower for ${ }^{6} \mathrm{He}$. Since the Coulomb potential decreases with distance, this is consistent with the barrier position being at a longer distance. In conclusion, the shift of the ${ }^{6} \mathrm{He}$ cross sections to higher values with respect to the quantities corresponding to ${ }^{4} \mathrm{He}$ systems is consistent with a static effect of the halo size, which "pushes" the barrier away to longer distances, at the same time lowering the barrier height.

Another big difference in the barrier parameters for the two projectiles can be seen in the barrier curvature parameter $\varepsilon_{0}$. The ratio of the respective values is $\varepsilon_{0}\left({ }^{6} \mathrm{He}\right) / \varepsilon_{0}\left({ }^{4} \mathrm{He}\right) \approx 2.5$. A large value of $\varepsilon_{0}$ (or $\hbar \omega_{0}$ ) implies a very narrow barrier. It can be seen from Wong's formula that $\varepsilon_{0}$ affects mainly the slope of the curve in the sub-barrier region. For larger energies, the two appearances of $\varepsilon_{0}$ in the formula tend to cancel each other, so there is no effect in this region. At sub-barrier energies, it has been shown that direct channels strongly couple with the elastic channel. This is a dynamic effect of the halo. Therefore, the large value of $\varepsilon_{0}$ obtained for the ${ }^{6} \mathrm{He}$ systems seems to be a signature for dynamic effects of the halo, which occur in the sub-barrier region. In this context, such dynamic effects would be simulated by an extremely narrow barrier. We must emphasize, however, that the respective barrier curvature is not expected to correspond to a realistic barrier resulting from the actual interplay of Coulomb and nuclear forces. Rather, it should be taken as a way to characterize the possible presence of dynamic effects within the simple framework of the one-dimensional barrier penetration model.

\section{Core-halo decoupling}

The fact that all these ${ }^{6} \mathrm{He}$ systems belong to a same trajectory in Fig. 4 implies that it is possible to scale among them, by just applying the proper transformations to energies and cross sections, in terms of the respective charges and masses (eq. 4.1). In principle, one could for example start with a point for some system in the curve, apply the transformations, and get what the cross section should be for a different system, at the corresponding energy. This line of reasoning lead to the idea of computing the reactions of the core of ${ }^{6} \mathrm{He}$ (or any halo nucleus), with a given target, by using the respective data for the plain core, in this case ${ }^{4} \mathrm{He}$. 


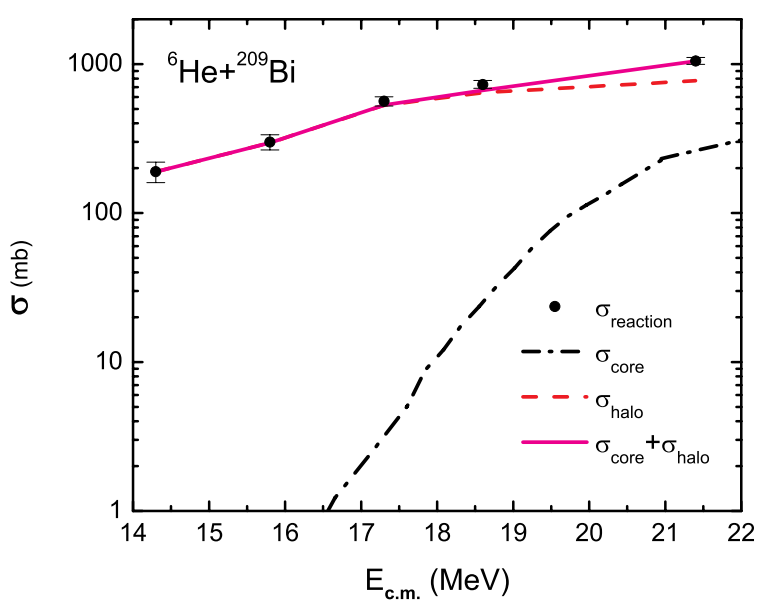

Figure 5: Additivity of cross sections for reactions of the core and reactions of the halo, for the ${ }^{6} \mathrm{He}+{ }^{209} \mathrm{Bi}$ system (taken from Ref. [6]).

To be more specific, by taking the particular system ${ }^{6} \mathrm{He}+{ }^{209} \mathrm{Bi}$, it is possible to deduce the reactions of the respective core out from the data for ${ }^{4} \mathrm{He}+{ }^{209} \mathrm{Bi}$ in the following way: any given value of $\sigma_{R}\left(\mathrm{E}_{c . m .}\right)$ for the latter system is first transformed into $\sigma_{R e d}\left(\mathrm{E}_{R e d}\right)$ through the relations of eq. 4.1 and then into the $\sigma_{R}\left(\mathrm{E}_{c . m}\right.$. $)$ value referred to the first system, by replacing the proper charges and masses in a second application of eq. 4.1. On the other hand, from the discussion in section 3 it is clear that the reactions with the halo are the one- and two-neutron transfer plus the breakup reactions. So, it is possible to sum the cross sections for the reactions of the core with those for the reactions of the halo, and compare to the total reaction cross sections. This has been done in Ref. [6], as shown in Fig. 5.

In Fig. 5 the reactions of the core are represented by the black dash-dotted curve, the reactions of the halo are described by the dashed line, and the sum is given by the red solid line. The sum describes quite well the data for the total reaction cross sections (black points). This additivity in the cross sections provides an evidence that core and halo decouple from each other, since it implies that there is no interference term.

Since the decoupling should be a characteristic feature of the halo state, one may expect that it should show up also in interactions with different targets. In order to corroborate this point, the reported data for the ${ }^{6} \mathrm{He}+{ }^{64} \mathrm{Zn}$ system $[20,21]$ were also analyzed in this context [5]. Fig. 6 shows the respective results. It can be seen that these data are also consistent with a core-halo decoupling.

The same kind of analysis was done for the proton halo system ${ }^{8} \mathrm{~B}+{ }^{58} \mathrm{Ni}$. Reaction cross section data for this and the respective core system, ${ }^{7} \mathrm{Be}+{ }^{58} \mathrm{Ni}$, have been reported recently [28]. The results of the analysis, which was originally done in the same reference, are displayed in Fig. 7. The reactions of the core are shown by the dash-dotted line. The reactions of the halo are just the breakup reactions. There is only one experimental point for this, so CDCC calculations were used to cover all energies (dashed curve). Once again, the additivity of cross sections holds. 


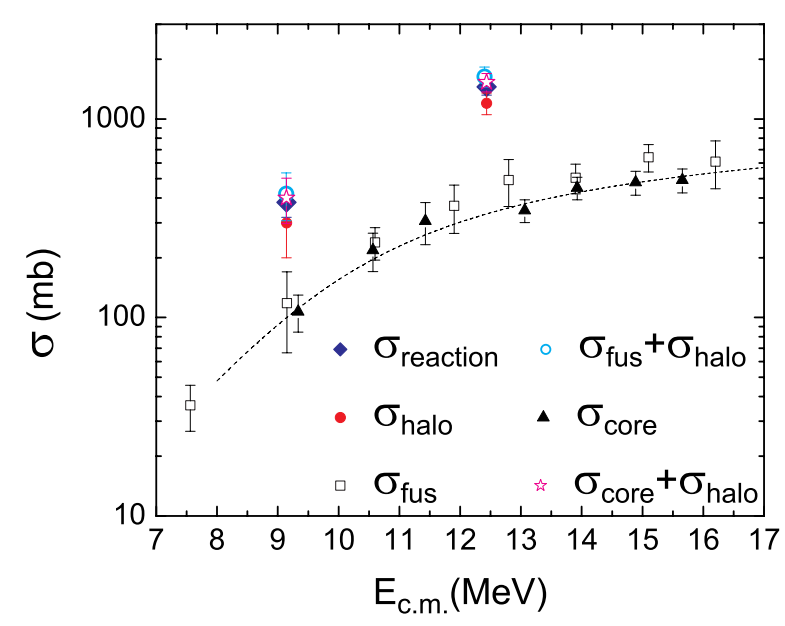

Figure 6: Additivity of cross sections for reactions of the core and reactions of the halo, for the ${ }^{6} \mathrm{He}+{ }^{64} \mathrm{Zn}$ system (taken from Ref. [5]).

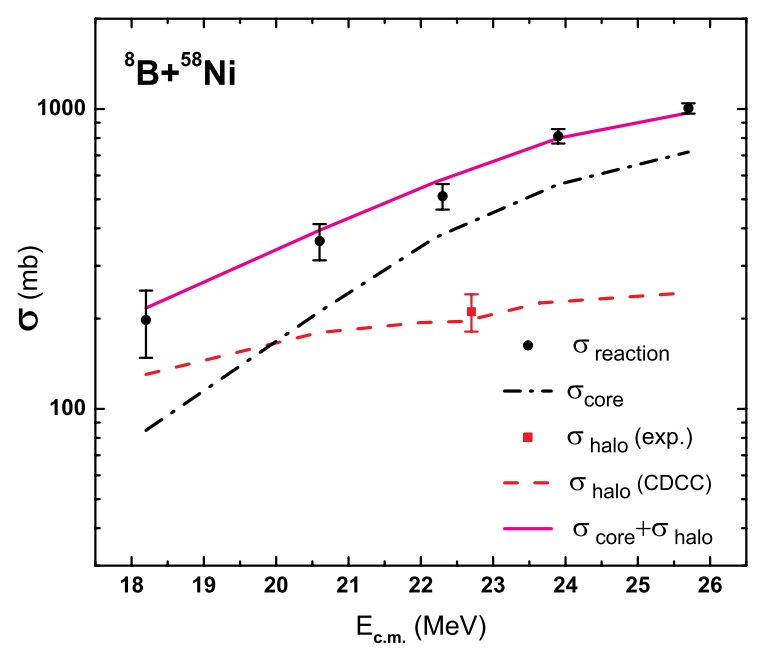

Figure 7: Additivity of cross sections for reactions of the core and reactions of the halo, for the ${ }^{8} \mathrm{~B}+{ }^{58} \mathrm{Ni}$ system (taken from Ref. [6]).

In order to show that the above core-halo decoupling is not just a trivial effect, it is worth looking at a system having ${ }^{17} \mathrm{O}$ as the projectile. With one neutron out from a closed shell, this nucleus can be considered as composed by an ${ }^{16} \mathrm{O}$ core, which is a double magic nucleus, plus a neutron. The binding energy of the valence neutron, $4.14 \mathrm{MeV}$, is well below the typical value for strongly bound nuclei. One may then say that ${ }^{17} \mathrm{O}$ shares some similarities with halo nuclei, and this makes it an interesting case study for comparison of halo versus non-halo features. Data for ${ }^{16,17} \mathrm{O}+{ }^{208} \mathrm{~Pb}$, reported in Ref. [30], were analyzed within this context in Ref. [6]. The results are shown in Fig. 8. It can be seen that the additivity property is not satisfied in this case. In other words, the decoupling does actually seem to be a characteristic feature of true halo systems. 


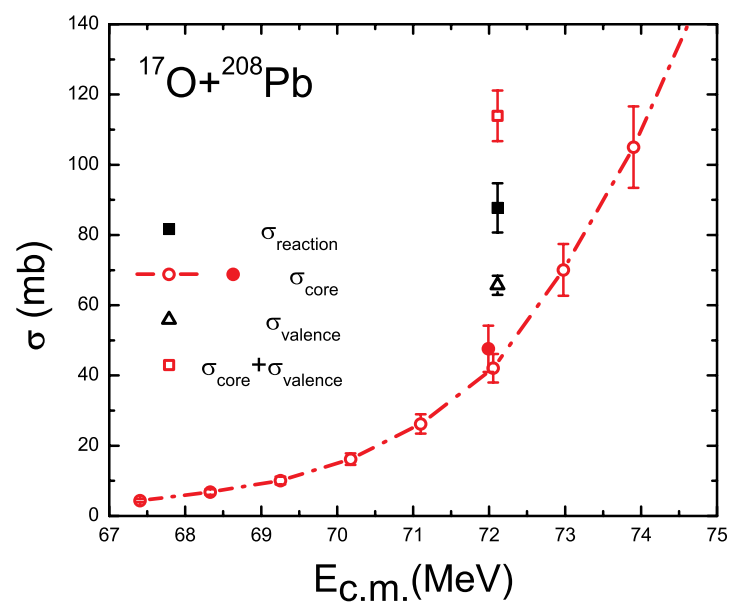

Figure 8: Total and quasielastic cross sections for ${ }^{17} \mathrm{O}$ and its core, on a ${ }^{208} \mathrm{~Pb}$ system. Data from Ref. [30]. (taken from Ref. [6]).

It has been shown theoretically [31], in the context of intermediate energy reactions, that good halo nuclei should approximately satisfy the general relation $\sigma_{R}($ halo state $)=\sigma_{- \text {halo }}+\sigma_{R}($ core $)$, where $\sigma_{- \text {halo }}$ is the halo removal cross section (see also Ref. [32]). The previous results are an experimental realization that this is true for the near Coulomb barrier region.

\section{Proton-halo effects}

While much work has been done on neutron-halo projectiles (see Table 1 and Refs. [2, 3, 4]), the present data on proton-halo systems are still scarce. The radioactive nucleus ${ }^{8} \mathrm{~B}$, adjacent to the proton drip line and having a very small proton separation energy $(0.138 \mathrm{MeV})$, has been recently studied in this context. In Ref. [33], an angular distribution for the breakup of ${ }^{8} \mathrm{~B}$ on a ${ }^{58} \mathrm{Ni}$ target was measured for a near barrier energy. It was found that Coulomb-nuclear interference at very large distances still plays an important role, thus providing evidence for the proton-halo nature of ${ }^{8} \mathrm{~B}$. More recently [28], elastic scattering measurements for the same system led to a total-reaction excitation function for near and sub-barrier energies. Most strikingly, the respective reduced curve follows the same path as the ${ }^{6} \mathrm{He}$ systems in Fig. 4, despite important differences in structure, binding energy and reaction mechanisms for these two projectiles. Clearly, a static halo effect similar to the one discussed in section 5 for ${ }^{6} \mathrm{He}$ is also present in the reaction cross sections of the proton-halo system.

While the large reaction cross section enhancement observed for neutron halo systems is mainly related to the corresponding neutron transfer channels (section 3), the breakup process gives the main direct channel in the case of the proton halo projectile [28]. In semiclassical terms, it would seem that in this case the effect of Coulomb polarization on the halo state tends to inhibit possible proton transfer channels. However, in contrast to the clear predominance of direct reactions for the case of neutron halo systems, the measured breakup cross section for ${ }^{8} \mathrm{~B}+{ }^{58} \mathrm{Ni}$, integrated over the whole solid angle, is still about $400 \mathrm{mb}$ short of saturating the corresponding 


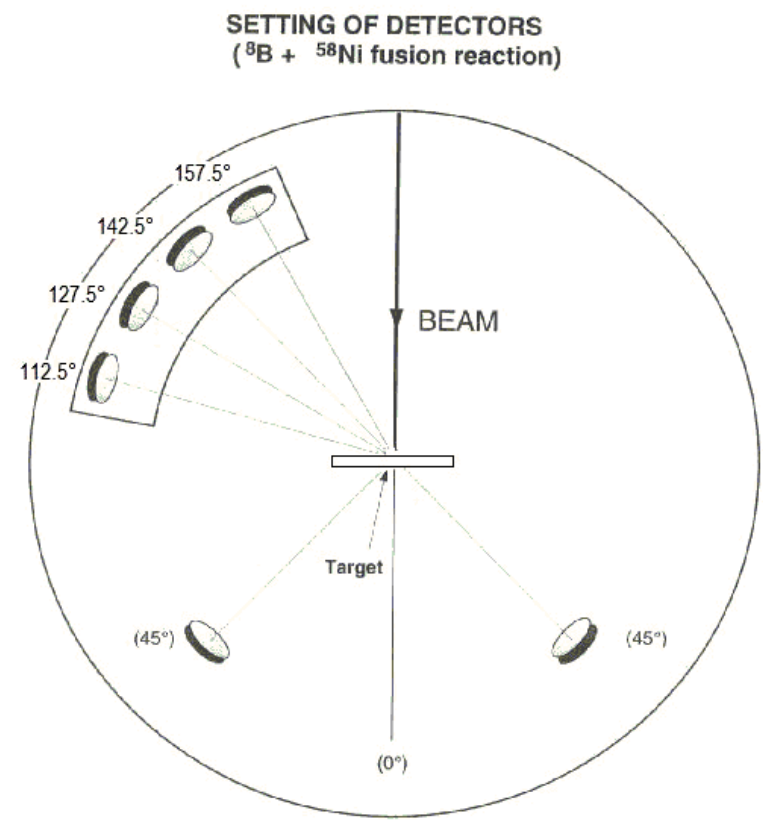

Figure 9: Experimental setup used in the fusion experiment.

total reaction cross section. So, one or more additional reaction processes must be present, with important contributions.

Recently, measurements for the fusion channel of the ${ }^{8} \mathrm{~B}+{ }^{58} \mathrm{Ni}$ system were reported [7]. Four telescopes were used at backward directions to measure the evaporated protons and two additional detectors at forward angles served to monitor the beam (Fig. 9). The proton yields were then converted to fusion cross sections by using the respective proton multiplicities calculated with a statistical model code. Nine energies were measured near and below the barrier and arguments were given to show that the reported cross sections can be reliably taken to correspond to total fusion.

The experimental results are shown in Fig. 10. A Wong function fit to the data yields the dash-dotted curve, with the indicated barrier parameters. The predictions for normal systems, on the other hand are represented by the solid curve. A large enhancement of the data with respect to the predictions is noticed. This enhancement shows up at all energies, similar to what was observed when comparing total cross section data for ${ }^{4} \mathrm{He}$ and ${ }^{6} \mathrm{He}$ systems (Fig. 4). As another similarity with that case, the fusion enhancement in the ${ }^{8} \mathrm{~B}+{ }^{58} \mathrm{Ni}$ system is also due to a big difference in the radius parameters for the two curves, as indicated in Fig. 10 (the respective ratio is $\mathrm{R}_{b}\left({ }^{8} \mathrm{~B}\right) / \mathrm{R}_{b}$ ("normal") $=1.26$ ). So, in the fusion data for the proton-halo system a strong static effect of the halo appears. This is in contrast, however, with the neutron-halo case, for which no static effect appears in the corresponding fusion data. This can be seen, for instance, from a comparison of figures 3 and 5. From the discussions in sections 3 and 6, the $\sigma_{f u s}$ points of Fig. 3 $\left({ }^{6} \mathrm{He}+{ }^{209} \mathrm{Bi}\right.$ ) should lie close to the $\sigma_{\text {core }}$ curve of Fig. 5 (see Fig. 3 in Ref. [6]), which corresponds to fusion data for ${ }^{4} \mathrm{He}+{ }^{209} \mathrm{Bi}$. In other words, no strong fusion enhancement with respect to normal 


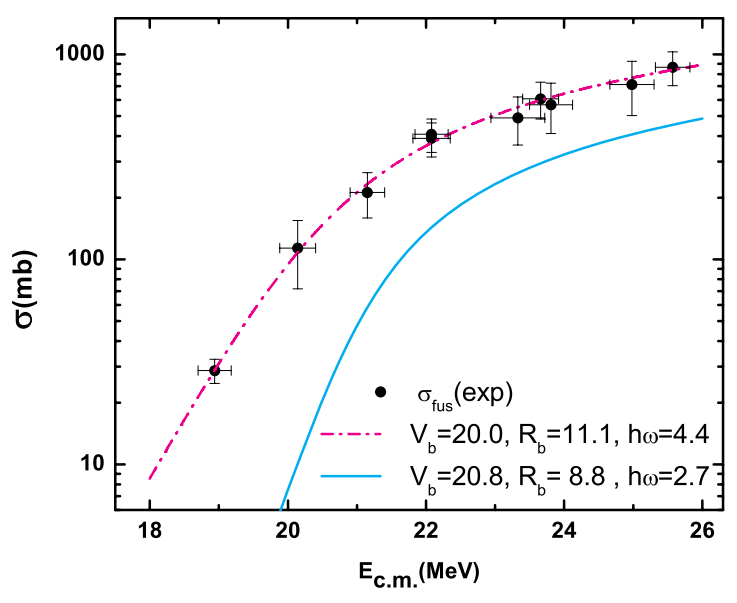

Figure 10: Fusion cross sections for the ${ }^{8} \mathrm{~B}+{ }^{58} \mathrm{Ni}$ system. The dash-dotted line is a Wong model fit to the data while the solid curve represents the predictions for normal systems. Data from Ref. [7].

systems appears in the neutron-halo case. This remarkable difference between proton- and neutronhalo systems might result from the different role played by Coulomb polarization in the case of a charged rather than a neutral halo.

\section{Conclusions}

A review of recent results for the neutron-halo projectile ${ }^{6} \mathrm{He}$ and the proton-halo projectile ${ }^{8} \mathrm{~B}$ is done. By considering measurements for a large variety of target nuclei within a wide energy region, it is seen that the total reaction cross sections for ${ }^{6} \mathrm{He}$ and its core, ${ }^{4} \mathrm{He}$, follow well defined trends when the data are properly reduced. By comparing the two trends, it is possible to characterize separately the static and the dynamic effects of the neutron halo. The scaling properties of the data could also be used to show that the reactions of the core decouple from the reactions of the halo, which turns out to be valid for both, neutron- and proton-halo systems. Finally, recent data for fusion of the ${ }^{8} \mathrm{~B}+{ }^{58} \mathrm{Ni}$ system show a remarkably strong static effect of the proton-halo. No equivalent effect is present in the fusion channel of the neutron-halo systems. This kind of effect for fusion has been seen in this system for the first time. It could indicate an important qualitative difference with the case of neutron halos, probably related to the different role played by Coulomb polarization in both cases.

\section{References}

[1] I. Tanihata et al., Measurements of interaction cross sections and radii of He isotopes,Phys. Lett. B160 (1985) 380.

[2] L. F. Canto, P. R. S. Gomes, R. Donangelo, and M. S. Hussein, Fusion and breakup of weakly bound nuclei, Phys. Rep. 424 (2006) 1. 
[3] N. Keeley, R. Raabe, N. Alamanos, and J. L. Sida, Fusion and direct reactions of halo nuclei at energies around the Coulomb barrier, Prog. Part. Nucl. Phys. 59 (2007) 579.

[4] N. Keeley, N. Alamanos, K. W. Kemper, and K. Rusek, Elastic scattering and reactions of light exotic beams, Prog. Part. Nucl. Phys. 63 (2009) 396.

[5] E. F. Aguilera, I. Martel, A. M. Sánchez-Benítez, and L. Acosta, Systematics of reactions with ${ }^{4,6} H e$ : Static and dynamic halo effects and evidence for core-halo decoupling, Phys. Rev. C 83 (2011) 021601(R).

[6] E. F. Aguilera, J. J. Kolata, and L. Acosta, Evidence for core-halo decoupling in halo systems, Phys. Rev. C 81 (2010) 011604(R).

[7] E. F. Aguilera et al., Near-Barrier Fusion of the ${ }^{8} B+{ }^{58}$ Ni Proton-Halo System, Phys. Rev. Lett. 107 (2011) 092701.

[8] J. J. Kolata et al., Sub-barrier Fusion of ${ }^{6} \mathrm{He}$ with ${ }^{209}$ Bi, Phys. Rev. Lett. 81 (1998) 4580.

[9] E. F. Aguilera et al., Transfer and/or Breakup Modes in the ${ }^{6} \mathrm{He}+{ }^{209}$ Bi Reaction near the Coulomb Barrier, Phys. Rev. Lett. 84 (2000) 5058.

[10] E. F. Aguilera et al., Elastic scattering and transfer in the ${ }^{6} \mathrm{He}+{ }^{209}$ Bi system below the Coulomb barrier, Phys. Rev. C 63 (2001) 061603(R).

[11] J. P. Bychowski et al., ${ }^{209} \mathrm{Bi}\left({ }^{6} \mathrm{He}, \alpha\right)$ reaction mechanisms studied near the Coulomb barrier using $n$ - $\alpha$ coincidence measurements,Phys. Lett. $\mathbf{B 5 9 6}$ (2004) 26.

[12] P. A. DeYoung et al., Two-neutron transfer in the ${ }^{6} \mathrm{He}+{ }^{209}$ Bi reaction near the Coulomb barrier, Phys. Rev. C 71 (2005) 051601(R).

[13] J. J. Kolata et al., Breakup of ${ }^{6}$ He incident on ${ }^{209}$ Bi near the Coulomb barrier, Phys. Rev. C 75 (2007) $031302(\mathrm{R})$.

[14] O.R. Kakuee et al., Elastic scattering of the halo nucleus ${ }^{6} \mathrm{He}$ from ${ }^{208} \mathrm{~Pb}$ above the Coulomb barrier, Nucl. Phys. A728 (2003) 339.

[15] A. M. Sánchez-Benítez et al., Study of the elastic scattering of ${ }^{6} \mathrm{He}$ on ${ }^{208} \mathrm{~Pb}$ at energies around the Coulomb barrier, Nucl. Phys. $\mathbf{A 8 0 3}$ (2008) 30.

[16] L. Acosta et al., Elastic scattering and $\alpha$-particle production in ${ }^{6} \mathrm{He}+{ }^{208} \mathrm{~Pb}$ collisions at $22 \mathrm{MeV}$, Phys. Rev. C 84 (2011) 044604.

[17] O.R. Kakuee et al., Long range absorption in the scattering of ${ }^{6} \mathrm{He}$ on ${ }^{208} \mathrm{~Pb}$ and ${ }^{197} \mathrm{Au}$ at $27 \mathrm{MeV}$, Nucl. Phys. A765 (2006) 294.

[18] P. N. de Faria et al., Elastic scattering and total reaction cross section of ${ }^{6} \mathrm{He}+{ }^{120}$ Sn, Phys. Rev. C 81 (2010) 044605.

[19] A. Chatterjee et al., In and $2 n$ Transfer With the Borromean Nucleus ${ }^{6}$ He Near the Coulomb Barrier, Phys. Rev. Lett. 101 (2008) 032701.

[20] A. Di Pietro, et al., Reactions induced by the halo nucleus ${ }^{6}$ He at energies around the Coulomb barrier, Phys. Rev. C 69 (2004) 044613.

[21] A. Di Pietro, et al., Structure effects on reaction mechanisms in collisions induced by radioactive ion beams, Phys. At. Nucl. 69 (2006) 1366.

[22] L. R. Gasques, et al., Experimental determination of the surface density for the ${ }^{6}$ He exotic nucleus, Phys. Rev. C 67 (2003) 024602; L. R. Gasques, private communication. 
[23] E. A. Benjamim, etal., Elastic scattering and total reaction cross section for the ${ }^{6} \mathrm{He}+{ }^{27}$ Al system, Phys. Lett. B647 (2007) 30.

[24] R. J. Smith, J. J. Kolata, K. Lamkin, A. Morsad, K. Ashktorab, F. D. Becchetti, J. A. Brown, J. W. Janecke, W. Z. Liu, and D. A. Roberts, Scattering of ${ }^{6} \mathrm{He}$ from ${ }^{\text {nat }} \mathrm{Ti},{ }^{27} \mathrm{Al}$, ${ }^{\text {nat }} \mathrm{C}$, and ${ }^{9} \mathrm{Be}$ at $\mathrm{E}=8-9$ MeV, Phys. Rev. C 43 (1991) 761.

[25] R. E. Warner, et al., Elastic scattering of $10 \mathrm{MeV}{ }^{6} \mathrm{He}$ from ${ }^{12} \mathrm{C}$, ${ }^{\text {nat }} \mathrm{Ni}$, and ${ }^{197} \mathrm{Au}$, Phys. Rev. C 51 (1995) 178.

[26] M. Milin, et al., The ${ }^{6}$ He scattering and reactions on ${ }^{12} \mathrm{C}$ and cluster states of ${ }^{14} \mathrm{C}, \mathrm{Nucl}$. Phys. A730(2004) 285.

[27] P. R. S. Gomes, J. Lubian, I. Padron, and R. M. Anjos, Uncertainties in the comparison of fusion and reaction cross sections of different systems involving weakly bound nuclei, Phys. Rev. C 71 (2005) 017601.

[28] E. F. Aguilera, et al., Reaction cross sections for ${ }^{8} \mathrm{~B},{ }^{7} \mathrm{Be}$ and ${ }^{6} \mathrm{Li}+{ }^{58} \mathrm{Ni}$ near the Coulomb barrier. Proton-halo effects, Phys. Rev. C 79 (2009) 021601(R).

[29] J. J. Kolata and E. F. Aguilera, Interaction barriers for light, weakly bound projectiles, Phys. Rev. C 79 (2009) 027603.

[30] J. S. Lilley, M. A. Nagarajan, D. W. Banes, B. R. Fulton, and I. J. Thompson, Multistep effects in reactions of ${ }^{17} \mathrm{O}$ with ${ }^{208} \mathrm{~Pb}$ near the coulomb barrier, Nucl. Phys. A463(1987) 710.

[31] K. Yabana, Y. Ogawa, Y. Suzuki, Reaction mechanism of ${ }^{11}$ Li at internediate energy, Nucl. Phys. A539 (1992) 295.

[32] K. Riisager, Nuclear Halos and Experiments to Probe Them, in The Euroschool Lectures on Physics with Exotic Beams, Vol. II, edited by J. Al-Khalili and E. Roeckl, Lect. Notes Phys. 700 (Springer, Berlin Heidelberg 2006), DOI 10.1007/b11743651 .

[33] V. Guimarães, et al., Nuclear and Coulomb Interaction in ${ }^{8}$ B Breakup at Sub-Coulomb Energies, Phys. Rev. Lett. 84 (2000) 1862. 